\title{
Noble-Metal-Free Copper Hydroxide as an Active and Robust Electrocatalyst for Water Oxidation at Weakly Basic pH
}

Shengsheng Cui, Xiang Liu, Zijun Sun, Pingwu Du*

CAS Key Laboratory of Materials for Energy Conversion, Department of Materials Science and Engineering, $i$ ChEM (Collaborative Innovation Center of Chemistry for Energy Materials), University of Science and Technology of China (USTC), Hefei, 230026, China

*Corresponding author: dupingwu@ustc.edu.cn

Tel/Fax: 86-551-63606207

\section{Contents}

Page S2, Figure S1. SEM images of as-synthesized $\mathrm{Cu}(\mathrm{OH})_{2}$ at loading amounts of (a) $0.2 \mathrm{mg} / \mathrm{cm}^{2}$, (b) $0.6 \mathrm{mg} / \mathrm{cm}^{2}$, (c) $0.8 \mathrm{mg} / \mathrm{cm}^{2}$, and (d) $1.0 \mathrm{mg} / \mathrm{cm}^{2}$.

Page S3, Figure S2. The Nyquist diagrams of $\mathrm{Cu}(\mathrm{OH})_{2} / \mathrm{FTO}$ electrode at an overpotential of $520 \mathrm{mV}$ in $0.1 \mathrm{M} \mathrm{KBi}$ at various loading amounts.

Page S4, Figure S3. SEM image of synthesized $\mathrm{Cu}(\mathrm{OH})_{2}$ after bulk electrolysis at 1.2 V for 6 hours.

Page S5, Figure S4. X-ray photoelectron spectroscopy (XPS) survey data (a) and high-resolution XPS spectra of Cu 2p (b); Cu LMM (c) and O 1s (d) of as-synthesized $\mathrm{Cu}(\mathrm{OH})_{2}$ material after bulk electrolysis in $1.0 \mathrm{M} \mathrm{KOH}$ solution.

Page S6, Table S1. Comparison of the OER activities of recently reported Cu-based catalysts. 

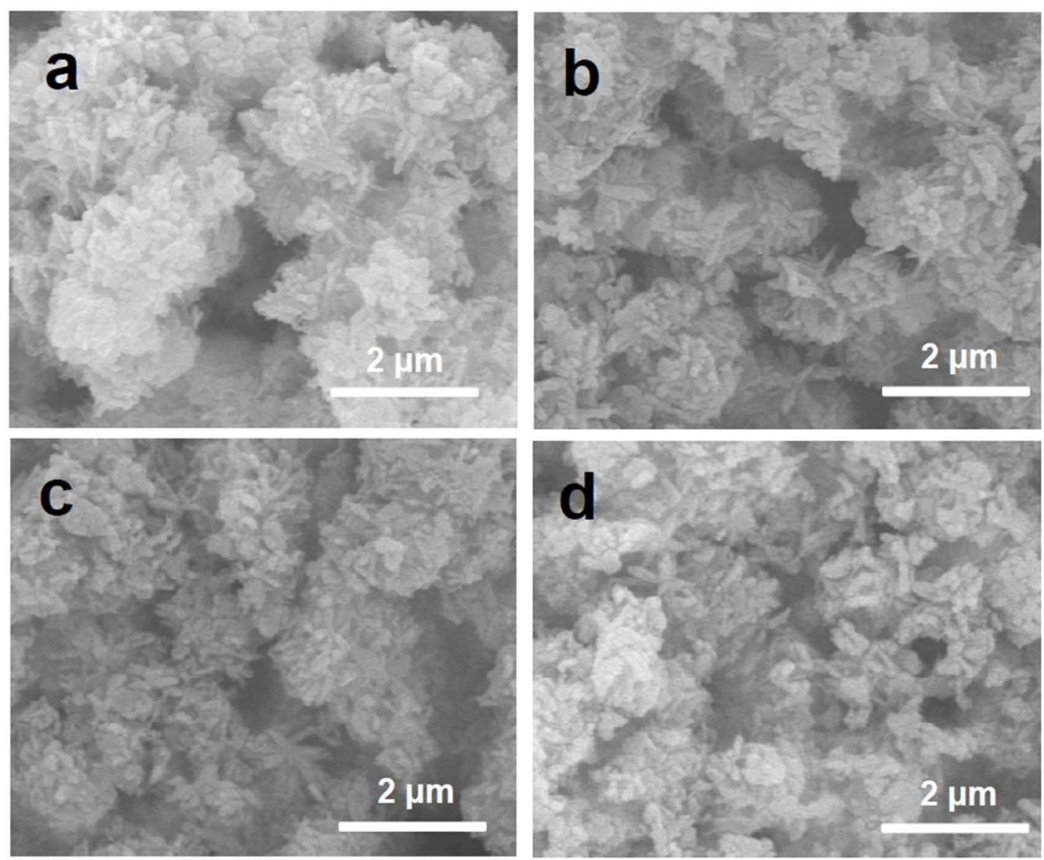

Figure S1. SEM images of as-synthesized $\mathrm{Cu}(\mathrm{OH})_{2}$ at loading amounts of (a) 0.2 $\mathrm{mg} / \mathrm{cm}^{2}$, (b) $0.6 \mathrm{mg} / \mathrm{cm}^{2}$, (c) $0.8 \mathrm{mg} / \mathrm{cm}^{2}$, and (d) $1.0 \mathrm{mg} / \mathrm{cm}^{2}$. 


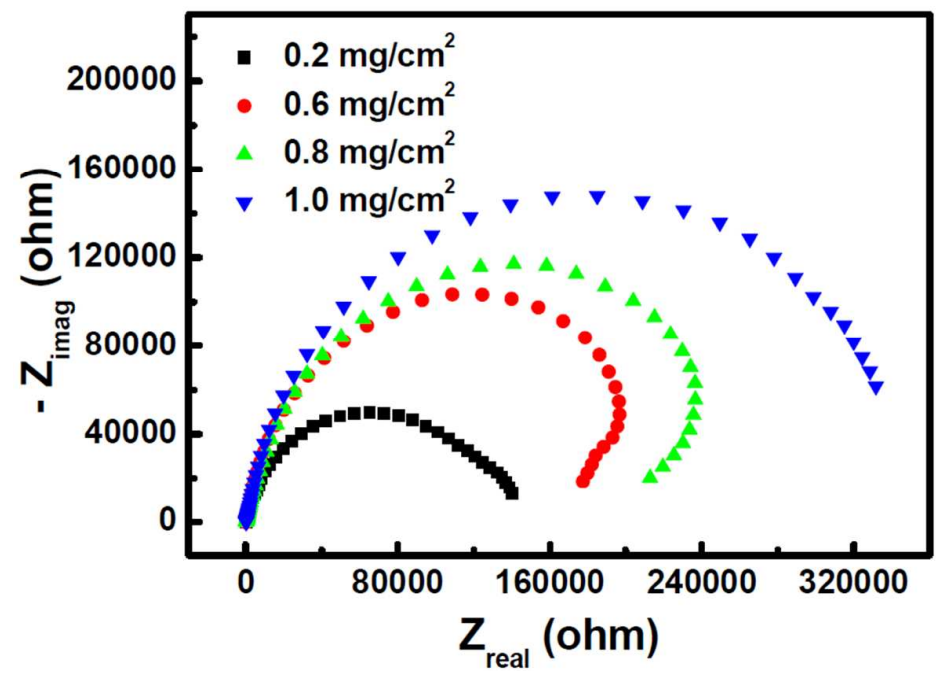

Figure S2. The Nyquist diagrams of $\mathrm{Cu}(\mathrm{OH})_{2} / \mathrm{FTO}$ electrode at an overpotential of $520 \mathrm{mV}$ in $0.1 \mathrm{M} \mathrm{KBi}$ at various loading amounts. 


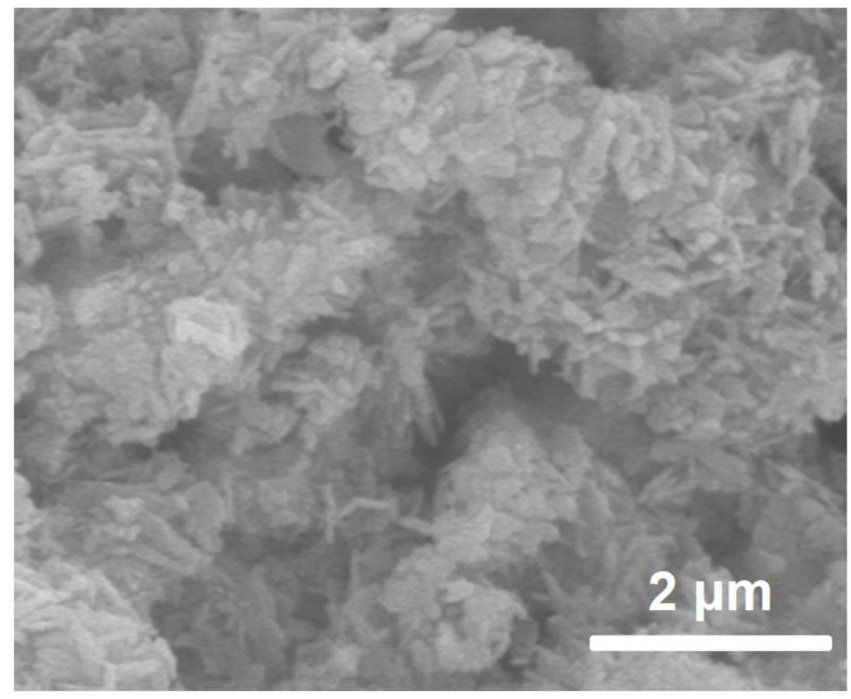

Figure S3. SEM image of synthesized $\mathrm{Cu}(\mathrm{OH})_{2}$ after bulk electrolysis at $1.2 \mathrm{~V}$ for 6 hours 

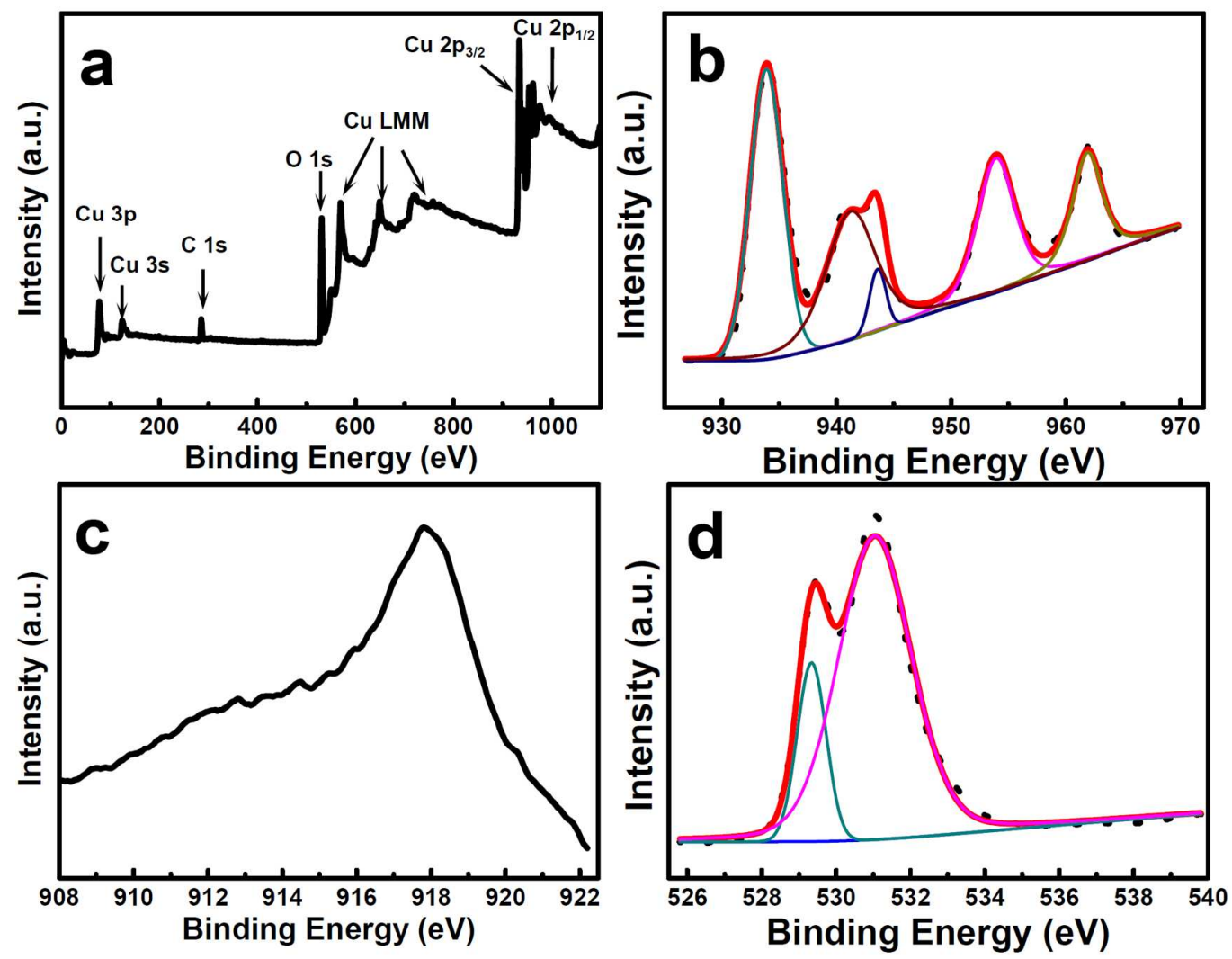

Figure S4. X-ray photoelectron spectroscopy (XPS) survey data (a) and high-resolution XPS spectra of $\mathrm{Cu} 2 \mathrm{p}(\mathrm{b}) ; \mathrm{Cu}$ LMM (c) and O 1s (d) of as-synthesized $\mathrm{Cu}(\mathrm{OH})_{2}$ material after bulk electrolysis in $1.0 \mathrm{M} \mathrm{KOH}$ solution. 
Table S1. Comparison of the OER activities of recently reported Cu-based catalysts.

\begin{tabular}{|c|c|c|c|c|}
\hline catalyst & $\eta(\mathrm{mV})^{\mathrm{a}}$ & $\begin{array}{c}\text { Tafel slope } \\
\text { (mV/dec) }\end{array}$ & References & System \\
\hline $\mathrm{Cu}^{\text {II }}$-bipyridine & $\sim 750$ & - & $\begin{array}{c}\text { Nat. Chem. 2012, 4, } \\
498 .\end{array}$ & \multirow{7}{*}{$\begin{array}{c}\text { Homogeneous } \\
\text { System }\end{array}$} \\
\hline$\left[\left(\mathrm{TGG}^{4-}\right) \mathrm{Cu}^{\mathrm{II}}-\mathrm{OH}_{2}\right]^{2-}$ & $\sim 520$ & - & $\begin{array}{c}\text { J. Am. Chem. Soc. } \\
\text { 2013, 135, } 2048 .\end{array}$ & \\
\hline $\begin{array}{c}{\left[\mathrm{Cu}_{2}(\mathrm{BPMAN})(\mu-\mathrm{OH})\right]^{3}} \\
+\end{array}$ & $\sim 780$ & - & $\begin{array}{l}\text { Angew. Chem. Int. } \\
\text { Ed. 2015, 54, } 4909 .\end{array}$ & \\
\hline $\mathrm{Cu}^{\mathrm{II}}$-LGr-ITO & $\sim 612$ & - & $\begin{array}{c}\text { ACS Appl. Mater. } \\
\text { Interfaces 2014, 6, } \\
18475 .\end{array}$ & \\
\hline $\mathrm{Cu}^{\mathrm{II}}$ & & & & \\
\hline $\begin{array}{c}-\mathrm{N}_{1}, \mathrm{~N}_{1}{ }^{\prime}-(1,2 \text {-phenylene }) \\
\text { bis }\left(\mathrm{N}_{2} \text {-methyloxalamid }\right. \\
\mathrm{e}\end{array}$ & $\sim 530$ & - & $\begin{array}{l}\text { J. Am. Chem. Soc. } \\
\text { 2015,137, 6758. }\end{array}$ & \\
\hline $\begin{array}{c}\mathrm{CuSO}_{4} \text { in } \mathrm{Na}_{2} \mathrm{CO}_{3} \\
\text { solution }\end{array}$ & $\sim 550$ & - & $\begin{array}{l}\text { Angew. Chem. Int. } \\
\text { Ed. 2013, 52, } 700 .\end{array}$ & \\
\hline Annealed $\mathrm{CuO}$ & $\sim 430$ & 61 & $\begin{array}{l}\text { J. Phys. Chem. C } \\
\text { 2016, 120, } 831 .\end{array}$ & \multirow{6}{*}{$\begin{array}{c}\text { Heterogeneous } \\
\text { System }\end{array}$} \\
\hline Cu-bifunctional & $\sim 510$ & 85 & $\begin{array}{c}\text { ACS Catal. 2015, 5, } \\
1530\end{array}$ & \\
\hline $\mathrm{CuO}$ from $\mathrm{Cu}-\mathrm{TEOA}$ & $\sim 490$ & 130 & $\begin{array}{c}\text { Inorg. Chem. 2015, } \\
\text { 54, } 3061 . \\
\text { Electrochem. }\end{array}$ & \\
\hline $\mathrm{CuO}$ from $\mathrm{Cu}-\mathrm{TPA}$ & $\sim 470$ & 56 & $\begin{array}{c}\text { Commun. 2014, 46, } \\
1\end{array}$ & \\
\hline $\mathrm{CuO}$ nanowires & $\sim 430$ & 54.5 & $\begin{array}{c}\text { Electrochimica Acta } \\
\text { 2015, 160, } 202 .\end{array}$ & \\
\hline $\mathrm{Cu}(\mathrm{OH})_{2}$ & $\sim 550$ & 78 & This work & \\
\hline
\end{tabular}

${ }^{a}$ For homogeneous system, the $\eta$ represents the onset overpotential, while it represents the required overpotential to reach a current density of $0.1 \mathrm{~mA} / \mathrm{cm}^{2}$ for heterogeneous system. 